\title{
Sous les identités sociales des internautes, les choix communicationnels, langagiers et stratégiques des entreprises?
}

L'exemple du site web institutionnel des firmes du CAC40

Under the social identities of Internet users, the communicational, language and strategic choices of the companies? The example of the institutional website in the CAC40 firms

\section{Gérald Lachaud}

\section{OpenEdition}

\section{Journals}

Édition électronique

URL : http://journals.openedition.org/communicationorganisation/5262

DOI : 10.4000/communicationorganisation. 5262

ISSN : 1775-3546

Éditeur

Presses universitaires de Bordeaux

Édition imprimée

Date de publication : 1 juin 2016

ISBN : 979-10-300-0059-7

ISSN : 1168-5549

\section{Référence électronique}

Gérald Lachaud, «Sous les identités sociales des internautes, les choix communicationnels, langagiers et stratégiques des entreprises ? ", Communication et organisation [En ligne], 49 | 2016, mis en ligne le 01 juin 2019, consulté le 03 janvier 2020. URL : http://journals.openedition.org/ communicationorganisation/5262 ; DOl : 10.4000/communicationorganisation.5262 


\section{Sous les identités sociales des internautes, les choix communicationnels, langagiers et stratégiques des entreprises? L'exemple du site web institutionnel des firmes du CAC40}

\section{Gérald Lachaud'}

La polyvalence du Web offre à la communication des entreprises une variété de réponses aux attentes de publics nombreux et divers. La catégorisation des internautes n'en devient que plus déterminante. Or, l'idéologie managériale a longtemps hésité sur les finalités d'un site web institutionnel d'entreprise. D'abord conçu comme une exigence de modernité et d'adaptation aux TIC, il est ensuite pensé comme un outil universel devant satisfaire l'ensemble des besoins d'une communication totale. Puis, il est considéré comme le symbole d'une communication transparente et authentique (Rouquette 256-261).

Mais pour une firme, l'utilisateur d'un site web n'est pas seulement un internaute.Il est aussi un acteur social aux multiples appartenances (actionnaire, investisseur, salarié, client, candidat, fan, voire opposant...). Les plans de communication, même lorsqu' ils ne relèvent pas d'une stricte raison marketing, s'emparent de ces appartenances comme autant de cibles (Libaert 153-162). Ainsi, la thématique de la RSE (Responsabilité Sociétale des Entreprises) identifie les principales parties prenantes concernées par l'activité des firmes (Capron \& Quairel-Lanoizelée 97-100). Le but est d'établir des relations contractuelles avec des partenaires, souvent issus du monde associatif, pour œuvrer à la résolution de problèmes de société (discrimination, changement climatique, biodiversité...). De même, les principes des « relations publiques généralisées » visent, " dans tous les domaines de la vie sociale, à fabriquer de l'adhésion ». Ce qui suppose de s'adresser non seulement " aux groupes ou aux audiences » mais aussi, et surtout, à des individus, des consommateurs, voire des citoyens (Miège 168). Le recours aux techniques de ciblage peut aussi

1 Gérald Lachaud est enseignant-chercheur à l'Université Jean-Moulin Lyon 3, membre d'ELICO Lyon 3. Ses travaux s'inscrivent dans une perspective sémiotique, communicationnelle et organisationnelle. Ils portent sur la communication numérique et sur la responsabilité sociétale des entreprises (RSE) ; gerald.lachaud@ univ-lyon3.fr 
signifier une volonté de maintenir "l'atomisation des individus et des groupes sociaux », afin d'empêcher l'émergence de significations concurrentes du sens global proposé par l'entreprise : "l'économie de marché et la concurrence économique comme fondement du lien social » (Le Moënne 113).

Par ailleurs, la généralisation des écrans mobiles contribue à transformer l'internaute en "smartphonaute ». Les concepteurs de sites web adaptent donc les contenus en ligne aux nouvelles conditions de consultation («web responsive " ou web adaptatif). Ce qui contribue à renforcer le " paradigme de l'expérience utilisateur » (Scapin, Senach, Trousse \& Pallot) dont nombre d'entreprises spécialisées se réclament pour justifier leur expertise dans l'ergonomie, l'attractivité et l'agencement des pages-écrans d'un site ou d'une application mobile (Boucher).

Ainsi, qu'ils soient imaginés ou réels, étayés par des mesures d'audience ou des études d'usages, issus de communautés plus ou moins virtuelles, créés ou renforcés par les technologies, les publics questionnent les professionnels de la communication. De même qu'ils suscitent l'intérêt des SIC (GimelloMesplomb \& Vilatte). Il convient donc de s'interroger sur les catégories que les entreprises privilégient dans leur politique de communication sur le web. Cette interrogation amène, au préalable, une réflexion sur les enjeux de la catégorisation des publics par les entreprises. Un corpus de page d'accueil permet, ensuite, d'examiner la place accordée à certaines appartenances sociales, de mesurer l'influence de l'activité de l'entreprise sur la définition même des identités sociales attribuées aux internautes et de souligner le poids des décisions organisationnelles et des contingences de politique générale.

\section{Méthodologie d'élaboration et de traitement du corpus}

Le corpus est composé de la page d'accueil du site Web institutionnel des quarante entreprises du CAC40. Les captures d'écran ont eu lieu en deux vagues de consultation, la première, les 26 et 27 janvier 2014 et le 3 février 2014, la seconde les 8 et 9 février 2015.

Les rubriques et les onglets du menu de chaque page d'accueil ont été répertoriés afin de lister les substantifs et les qualificatifs qui permettent à l'internaute de reconnaître son appartenance à une catégorie sociale. Seuls les termes qui nomment une personne ont été retenus ( Actionnaire », "Client », "Journaliste »...). Les adjectifs qui caractérisent une qualité humaine (« être engagé et responsable ») ont été exclus. L'acronyme ISNA (Identité Sociale Numérique Affichée) désigne toute rubrique de la page d'accueil qui remplit les conditions énoncées. L'ensemble des ISNA et des sèmes associés (autres substantifs, qualificatifs, pronoms...) a fait l'objet d'un dénombrement et d'une analyse selon les différentes catégories identitaires possibles (genre, statut économique ou social...). 


\section{La catégorisation des publics: entre médiation marketing et stratégie de communication}

Dans leur volonté de répondre de manière efficace à une demande considérée comme solvable, les entreprises s'appuient sur un indispensable travail de médiation marchande (Cochoy 1999). Une partie essentielle de cette médiation est assurée par le marketing. Dès ses origines, sa mission consiste, entre autres, à attribuer aux individus des dispositions, sociales, psychologiques et économiques qui, une fois jugées pertinentes et représentatives, seront incorporées dans des dispositifs (services ou produits) mis sur le marché (Cochoy 2004 : 18-32). Des catégories sociales comme celle d'usager, d'utilisateur, de consommateur, et surtout de client, vont alors être mobilisées pour naturaliser et rendre opératoires les dispositions tant recherchées. Le travail de catégorisation des publics et des cibles peut donc s'inscrire dans une vision rationnelle de la stratégie des entreprises. Face à la concurrence, les firmes analysent les contraintes et les ressources. Elles tirent ensuite avantage de la situation par un positionnement destiné à valoriser leurs compétences et leurs atouts auprès de clients, comme de partenaires, identifiés et désignés avec clarté. Ce processus repose sur une maîtrise de l'information, une objectivation des choix et une planification des actions. Cependant, les sciences de gestion elles-mêmes admettent une autre acception de la stratégie, fondée sur une vision plus incrémentale et circonstancielle de la rationalité. Les entreprises sont alors considérées comme une instance engagée dans une suite d'événements plus ou moins lisibles et soumise à des jeux d'acteurs aux intérêts divergents qui pèsent sur les orientations choisies in fine (Gervais \& Herriau 9-10).

Or,unestratégiede communication peuts'appuyersurde multiplestechniques de ciblage et de nombreuses typologies. Mais toute catégorisation hermétique se heurte aussi à la complexité et surtout à la labilité des publics (Libaert \& de Marco 84). D'autant que les analystes ont souligné le caractère antagoniste ou contradictoire des dispositions prêtées aux catégories sociales (usager, citoyen, consommateur ou client) invoquées pour légitimer de nombreuses décisions gestionnaires et managériales (Chauvière \& Godbout, Vitalis). De plus, les dimensions sensible et symbolique de la communication sont de plus en plus prises en compte, notamment par le marketing expérientiel (Hetzel). La médiation marketing s'appuierait, donc, autant sur la reconnaissance d'un état sémiotique variable et polymorphe (Legris-Desportes) que sur la valorisation d'un statut identitaire figé et affirmé. D'autant que, désormais, les dispositifs de communication numérique permettent d'associer la seule figure du client avec des dispositions variées de concepteur, d'évaluateur, de producteur, voire de citoyen (Bocquet \& Lachaud).

Cependant, toute inventivité conceptuelle doit aussi composer avec les contraintes langagières et institutionnelles. En effet, les catégories sociales et sémantiques compatibles avec la médiation marketing sont en nombre limité. 
De plus, leur pertinence varie selon les espaces où elles sont discutées et évaluées. Ainsi, la célèbre "ménagère de moins de cinquante ans » a connu une notoriété notable dans les milieux du marketing et de la mesure d'audience. À charge pour les services et agences de communication de transformer, avec plus ou moins de réussite, cette notion en une figure rhétorique et esthétique du discours commercial et publicitaire des entreprises.

Il semblerait que le corpus étudié porte trace, en partie, de tels enjeux.

\section{L'emprise de la communication financière: s'adresser aux actionnaires et aux investisseurs}

Les entreprises du CAC40 qui identifient les internautes et affichent au moins une appartenance sociale dans les rubriques de la page d'accueil de leur site web institutionnel sont majoritaires. Elles sont vingt-huit en 2015, pour 96 ISNA au total. Elles étaient vingt-sept en 2014, pour 77 ISNA. Plusieurs firmes ont ajouté quelques ISNA à leur page d'accueil (Bouygues, Sanofi...). Certaines adoptent en 2015 le principe même de l'identification sociale des internautes (Michelin, Renault, Vallourec). Deux l'ont abandonné (Legrand, Safran).

Cependant, une page d'accueil sans ISNA ne signifie pas un refus de communiquer envers une catégorie sociale. Ainsi, douze entreprises s'adressent aux actionnaires et aux investisseurs par la rubrique "Finance ». Vingt choisissent les rubriques "Presse ", " Médias » ou " Espace presse " plutôt que « Journalistes ». Trente affichent les rubriques «Talents », " Carrière(s) », «Recrutement » pour attirer les candidats à l'embauche. Enfin, le développement de sites marchands pour les clients, de l'Intranet pour les collaborateurs et de sites orientés vers la « marque employeur » (Ryan, Gubern, Rodriguez) pour les jeunes diplômés explique aussi l'absence de certaines identités sociales sur un site institutionnel.

Six catégories sociales regroupent $8 / 10^{\mathrm{e}}$ des 96 ISNA mentionnées en 2015 : «Investisseur(s) », « Actionnaire(s) », " Candidat(s) », « Journaliste(s) », "Client(s) », "Fournisseur(s) ». Mais les catégories " Actionnaire(s) » et «Investisseur(s) » apparaissent deux à trois plus que les autres. Elles représentent plus de $4 / 10^{\mathrm{e}} \mathrm{du}$ corpus $(43,75 \%)$, une proportion légèrement inférieure à celle de $2014(45,45 \%)$.

Enfin, le genre féminin, au pluriel ou au singulier, n'est jamais proposé à l'internaute, dans aucune des catégories recensées.

\begin{tabular}{|l|c|c|c|c|}
\hline \multicolumn{5}{|c|}{ Nombre et répartition des ISNA } \\
\hline & \multicolumn{2}{|c|}{2014} & \multicolumn{2}{c|}{2015} \\
\hline ISNA & $\mathrm{Nbr}$ & $\%$ & $\mathrm{Nbr}$ & $\%$ \\
\hline Investisseur(s) & 21 & 27,27 & 24 & 25 \\
\hline Actionnaire(s) & 14 & 18,18 & 18 & 18,75 \\
\hline
\end{tabular}




\begin{tabular}{|l|c|c|c|c|}
\hline & \multicolumn{2}{|c|}{2014} & \multicolumn{2}{c|}{2015} \\
\hline Candidat(s) & 7 & 9,1 & 12 & 12,5 \\
\hline Journaliste(s) & 7 & 9,1 & 10 & 10,47 \\
\hline Client(s) & 6 & 7,8 & 6 & 6,25 \\
\hline Fournisseur(s) & 5 & 6,5 & 7 & 7,29 \\
\hline Autres & 17 & 22,01 & 19 & 19,79 \\
\hline Total & 77 & 100 & 96 & 100 \\
\hline Nombre de site & 27 & & 28 \\
\hline
\end{tabular}

Source: auteur

Au regard des identités sociales visibles sur la page d'accueil, la communication institutionnelle des entreprises semble donc privilégier les objectifs et les cibles de la communication financière. Les préconisations des professionnels qui affirment à l'envi l'importance de " prendre en compte tous les publics de l'entreprise » (Hurel du Campart \& AACC Corporate 78-81) paraissent quelque peu oubliées. Il est vrai, aussi, que la communication économique et financière est devenue depuis une décennie une nécessité pour les entreprises face au renforcement des autorités de régulation des marchés financiers, à la mondialisation des places boursières et à l'essor des législations sur le gouvernement d'entreprise (Léger 12-22).

Mais la communication financière s'intéresse parfois aux internautes en fonction des aléas vécus par l'entreprise. La page d'accueil du site de Vivendi semble en témoigner.

\section{L'identité attribuée aux internautes: un enjeu de remémoration organisationnelle?}

Quatorze firmes affichent une ISNA «Actionnaire(s) » en 2014 ou en 2015 sur leur page d'accueil. Seules deux ajoutent le qualificatif « individuel(s)». Michelin le fait en 2015 dans le cadre d'une refonte générale de sa page d'accueil et de l'adoption du principe des ISNA. En revanche, Vivendi opte pour ce choix, en remplacement de la rubrique «Finance », dès l'année 2012, toujours à l'occasion d'une refonte générale de la page d'accueil. Les Archives de l'internet permettent de situer ce changement entre le $1^{\text {er }}$ septembre et le 17 décembre.

Or, l'expression « actionnaire(s) individuel(s) » constitue presque un pléonasme. En effet, pour la communication financière, un « actionnaire " est un particulier qui place son argent en bourse à titre individuel. Il s'oppose à un " investisseur " qui agit pour le compte d'une institution financière (banque, société d'assurance, fonds de placement...).

Le choix de Vivendi est-il dû au hasard ? L'histoire récente de cette entreprise incite à penser le contraire. Née en 1998 d'une série de fusions et acquisitions, Vivendi se heurte très vite à des difficultés insurmontables : 
endettement, pertes cumulées et menace de banqueroute. Son emblématique PDG, Jean-Marie Messier, démissionne en 2002. La même année, son successeur, Jean-René Fourtou, vends de nombreux actifs pour rembourser les emprunts et équilibrer les comptes. Des «petits porteurs " s'estiment lésés. Ils déposent plainte en France et aux États-Unis. Depuis, la vie du groupe est rythmée par plusieurs procès, en première instance en 2010 et 2011, en appel en 2013 et 2014. Cette chronique judiciaire se double d'un feuilleton entrepreneurial pour le contrôle du capital. Vincent Bolloré devient actionnaire principal, avec un peu plus de $5 \%$ des actions, le 16 octobre 2012. En désaccord avec les décisions de Jean-Louis Fourtou, il obtient son départ au bout de deux ans de lutte, le 24 juin 2014.

Ainsi, la refonte de la page d'accueil du site web institutionnel intervient à la fin de l'année 2012, lors de la conjonction de trois phénomènes. Le premier est la temporalité de la justice, qui met à l'agenda médiatique le litige entre Vivendi et certains actionnaires. Le deuxième est la lutte de pouvoir au sommet hiérarchique d'une entreprise dont la faible concentration capitalistique autorise un raid boursier. Le dernier est l'importance croissante du Web et des outils numériques dans les actions de communication institutionnelle et de relations publiques. Ce qui était loin d'être le cas en 2002, lors des premiers dépôts de plainte des associations de " petits porteurs ».

L'apparition de la rubrique pléonastique "Actionnaires individuels » fin 2012 n'est donc pas seulement le signe de l'intensité de la crise qui affecte Vivendi depuis 2002. Elle est aussi la trace d'une appropriation du passé. En effet, les événements vécus par l'entreprise font l'objet d'un traitement informationnel pour alimenter le stock de connaissances conservées, préservées et gérées par les systèmes documentaires (Stein). Mais les péripéties subies par les firmes se prêtent aussi à une action de "remémoration organisationnelle ». Ce processus ne se réduit pas à une simple opération technique. Il est avant tout une pratique « située et incorporée » au sein d'une communauté dont les buts doivent faire sens pour les membres : "In other words, organizational remembering as a practice is not a means of storage, but a process that actively constructs meaning " (Feldman \& Feldman 870). La " remémoration organisationnelle " est donc constituée, et pas seulement influencée, par différents facteurs contextuels. Certains relèvent d'un niveau individuel : les jugements intellectuels et moraux des personnes porteuses d'un passé et d'émotions. D'autres relèvent d'un niveau collectif : les forces économiques et politiques ainsi que la culture et l'historicité de l'organisation. $\mathrm{La}$ " remémoration organisationnelle » participe d'un mouvement plus large de «sens-making » qui permet de re-construire les faits antérieurs pour qu'ils fassent sens et soient "énactés » dans la situation présente vécue au sein du collectif de travail (Weick 7-29).

C'est donc en 2012, dans un contexte médiatique, juridique et managérial particulier que la relation de Vivendi avec ses actionnaires, dégradée depuis 
2002, prend une autre signification. Remémorés et actualisés, les événements sont sans doute crédités d'une acception inédite qui se traduit par une nouvelle rubrique pléonastique. La prochaine clôture du chapitre judiciaire de l'histoire de Vivendi par la Cour de cassation verra, peut-être, la disparition d'une expression redevenue incongrue au regard des habitudes langagières des firmes du CAC40. Rien de tel nétait toutefois constaté début 2016.

Cependant, la communication institutionnelle s'adresse à d'autres d'internautes que les actionnaires et les investisseurs. L'exemple du terme "Client ", ainsi qu'une certaine forme de déférence et de politesse employée par les banques, semblent montrer, dans ces cas-là, l'influence du secteur d'activité.

\section{L'influence de l'activité des entreprises sur les relations aux internautes}

En 2014, six firmes affichent une rubrique « Clients » : Capgemini, Carrefour, Crédit Agricole, Lafarge, Legrand et Société Générale. En 2015, Lafarge et Société Générale délaissent cette catégorie. Mais Michelin et Veolia s'adressent désormais à elle. Cette récurrence est surprenante. En effet, un site marchand paraît plus approprié pour communiquer avec les clients. Une telle ISNA pourrait donc signifier la persistance d'une stratégie de communication globale qui tendait, à lorigine, à faire du site web institutionnel un portail pour l'ensemble des publics de lentreprise. Crédit Agricole semble être encore dans ce cas puisque l'ISNA "Clients » renvoie à un espace privé sécurisé, de nature marchande.

Cependant, les firmes qui opèrent sur un marché destiné aux entreprises $(\mathrm{BtB})$ ont une autre acception du terme "clients ». Associé à un substantif, il devient un procédé argumentaire destiné à témoigner du sérieux et de la réputation de l'entreprise. Ainsi, Legrand propose en 2014 une rubrique "Clients export " pour montrer son dynamisme international. Sans doute jugée ambiguë, cette rubrique est rebaptisée en 2015 « Nos produits à l'international ». Mais pour une entreprise de conseil en informatique, où les enjeux de sécurité et de confiance sont importants, la parole d'anciens clients possède à l'évidence une valeur testimoniale inestimable. Capgemini pérennise donc, en 2014 comme en 2015, la rubrique " Témoignages clients ».

La nature de l'activité semble même impacter la façon de communiquer auprès des internautes.

En effet, la page d'accueil de BNP Paribas, Crédit Agricole et Société Générale propose à l'internaute de choisir, selon le cas, entre différentes appartenances : " Actionnaire(s) », « Investisseur(s) ", « Journaliste ", " Candidat », " Client », "Fournisseur ", " Particulier ». Or, les trois sites continuent, en 2014 et en 2015, d'afficher au-dessus de ces appartenances une question "Vous êtes : ». La consultation des Archives de l'internet montre que d'autres entreprises du CAC40 (comme EDF en avril 2010 ou Pernod-Ricard en novembre 2011) posaient elles aussi cette question aux internautes. Depuis, toutes ont cessé de le faire, à l'exception de L'Oréal. Par ailleurs, deux autres firmes renouent, en 2015, 
avec l'invite adressée aux internautes. Renault affiche une simple proposition (« votre profil : ), quand Michelin préfere une suggestion (" choisissez votre profil»).

Pourquoi, dès lors, BNP Paribas, Crédit Agricole et Société Générale restent-elles unanimes pour maintenir une pratique éditoriale jugée désuète par l'immense majorité des autres firmes du CAC40 ? Leur refus du terme " profil » est-il dû à une connotation jugée froide et distante, généralement associée en informatique à la notion d'utilisateur ? L'importance de la relation de service dans la culture organisationnelle du secteur bancaire pourrait être un élément d'explication. Les réflexions pionnières proposées par la sociologie interactionniste ont montré qu'un prestataire assure d'autant mieux la partie technique et la partie contractuelle de la relation de service lorsqu'il maîtrise, par sa compétence sociale, la partie relationnelle, fondée sur les marques de civilité, de politesse et de reconnaissance échangées avec le bénéficiaire de la prestation (Goffman 380-383). Or, dans le secteur bancaire, la relation de service prend souvent la forme d'un conseil intuitu persone, c'est-à-dire conçu et formulé selon les caractéristiques particulières des individus. Certes, ce secteur n'a pas l'exclusivité de l'obligation de conseil personnalisé. Mais la loi et la jurisprudence en rendent l'application plus impérative et sanctionnent plus souvent tout manquement. Les procès intentés par un client mécontent des résultats d'un placement suggéré par son banquier ne sont pas rares. Rien de comparable ne peut être noté pour les agences de conseil en communication (comme Publicis) ou les sociétés de services en ingénierie et informatique (comme Capgemini).

La présence d'une question de civilité et de politesse «Vous êtes » dépasse donc, sans doute, la seule volonté d'orienter l'internaute. Elle semble illustrer la prépondérance d'une des trois préoccupations managériales à l'égard du web. Ces préoccupations ont été soulignées par la presse économique et la presse professionnelle (Rouquette 280-281). Elles sont portées par des métiers et des services qui défendent, selon des principes de justification différents, leur vision du web. Ainsi, le service communication insiste sur l'obligation de cohérence, sur le fond et sur la forme, du site web avec l'identité globale de l'entreprise. Le service informatique met l'accent sur la modernisation et la standardisation des interfaces et des plates-formes qui servent de support technologique aux contenus. Le service marketing souligne la nécessité d'adapter les contenus à la logique d'individualisation de la relation client.

Le probable conflit d'interprétation entre ces différents services s'est-il traduit, pour l'ensemble du secteur bancaire, par une discussion formelle sur le maintien, ou non, d'un « signe passeur » (Davallon \& Jeanneret 50) jugé désuet par les autres entreprises du CAC40 ? Linfluence du service marketing et de la culture de la relation client a-t-elle pesé dans la décision ? Il est difficile de répondre à ces questions. Mais force est de constater que, chez EDF, la formule « vous êtes », présente en 2010, est remplacée à partir de 2012 par une 
expression ("accès direct ») qui paraît plus mâtinée de langage informatique que marketing (source : les Archives de l'internet).

Cependant, l'étude des pages d'accueil laisse aussi entrevoir la prégnance d'instances décisionnelles préoccupées par d'autres considérations.

\section{Une mise en visibilité stratégique des salariés sur le web?}

L'absence d'une rubrique consacrée aux salariés sur les pages d'accueil du corpus ne laisse d'étonner. Certes, la communication interne privilégie l'Intranet. Mais les entreprises n'auraient-elles aucun discours public à tenir sur celles et ceux qui sont aussi la force et l'âme de l'organisation ? Un tel silence pourrait confiner au mépris, voire au déni de reconnaissance (Honneth 262). À l'inverse, il pourrait aussi résulter d'une volonté d'éviter toute critique contre une forme de « reconnaissance stratégique » dont les buts et les objectifs sont contraires à un acte réel de reconnaissance sociale fondée sur l'autonomie et la réalisation du sujet (Heller 94-95).

Seules deux firmes du CAC40 qui s'adressent à leurs " collaborateurs " permettent d'approfondir la question : Carrefour et Pernod Ricard.

À partir de la fin de l'année 2013, comme en attestent les Archives de l'internet, Carrefour décline les rubriques principales de la page d'accueil selon le parti-pris d'une énonciation collective : «Nos magasins », « Nos produits », « Nos performances ». Les salariés du groupe n’échappent pas à la règle avec la rubrique "Nos collaborateurs ». La mise en place de cette nouvelle page d'accueil intervient un peu plus d'un an après le départ inattendu du PDG Lars Olofsson, remercié pour résultats insuffisants et choix stratégiques erronés. Son successeur, Georges Plassat, a la réputation dêtre un sauveur charismatique, dur et exigeant, mais capable de redresser les situations difficiles. Surtout, il est considéré comme un spécialiste du secteur, un homme de terrain qui connaît les métiers de la grande distribution et qui mesure l'importance de l'engagement des salariés (Meignan). En 2013, il restructure la logistique et réorganise les magasins. À partir de 2014, il annonce l'évolution du marketing et du commercial vers le numérique mobile (Revue du digital). Or, début 2015, une fois sans doute révolue la phase de mobilisation interne consécutive à ces changements de politique générale, la page d'accueil du site institutionnel est modifiée. La rubrique " Nos collaborateurs » disparaît de l'écran.

Il convient, dans ces conditions, d'examiner l'hypothèse d'une homologie entre deux mises en visibilité des salariés de l'entreprise. La première, interne, serait opérée par la communication managériale chargée des relations interpersonnelles dans les situations de travail. Elle serait un acte de reconnaissance intéressée visant l'accroissement de la performance (Heller 103). Elle reposerait sur les différents «récits de la maisonnée» qui tentent d'impliquer, entre autres, les salariés et de les inclure, par le développement d'un sentiment d'appartenance, dans un collectif 
(d'Almeida150). En situation de crise, telle que celle vécue par Carrefour, ce collectif pourrait être valorisé en tant qu'équipe plutôt que groupe. Ce dernier est, en effet, « présenté et utilisé comme l'expression d'une subjectivité critique, voire contestataire, à l'intérieur d'une institution ». Alors que l'équipe " apparaît comme un collectif qui résulte d'une "mise en conformité" " (Gardère \& Gramaccia 107). Cependant, seule une consultation de l'Intranet (par exemple) permettrait de valider cette hypothèse.

En parallèle ou consécutivement à cette mobilisation interne, la communication institutionnelle procéderait à une mise en visibilité externe des salariés, notamment sur le web. Ce que la présence temporaire de la rubrique « Nos collaborateurs » dans notre corpus pourrait accréditer. Cette mise en visibilité externe constituerait avant tout « un vecteur de diffusion de critères de reconnaissance et une invitation, une incitation ou une pression à se reconnaitre dans ces critères " (Heller 103). Ce processus serait d'autant plus prégnant et engageant qu'il est publicisé et public (Bernard 90-91). Le contenu du menu déroulant de la rubrique "Nos collaborateurs " tendrait à confirmer ce point de vue. En effet, deux sous-rubriques illustrent le choix stratégique d'un recentrage sur le cœur de métier du groupe Carrefour : « La passion du commerce » et « Favoriser la confiance des collaborateurs ». Deux autres réaffirment publiquement la mobilisation collective : «Nos priorités » et «Nos engagements ». Mais l'enjeu serait, ici, moins la recherche de la performance des salariés que la production d'une subjectivité, considéré comme un assujettissement (Heller 103). Aussi, du point de vue de la communication organisationnelle, cette refonte générale de la page d'accueil du site de Carrefour mobilise, pour l'essentiel, des " actes d'engagement ", qui visent, entre autres, la coopération des individus. Cependant, il n'est pas interdit de voir dans ce travail langagier de l'énonciateur l'horizon d'attente des "actes de constat", qui « tirent leur force illocutoire d'un accord duel ou collectif sur un problème manifeste »(Gramaccia, 238-239).

La mise en visibilité d'un collectif de travail peut-elle aller jusqu'à la réification langagière des personnes ? Par son "énonciation de majesté " («Nos collaborateurs») Carrefour franchit peut-être le pas. Cependant, la firme n'applique pas ce traitement aux autres appartenances sociales affichées dans les rubriques de la page d'accueil. En effet, les termes "Actionnaire ", " Journaliste » et "Client », en 2015, auxquels étaient adjoints, en 2014, «Investisseur » et " Candidat » ne sont précédés d'aucun pronom ni article. Il est vrai, aussi, que toutes ces identités se situent d'un point de vue graphique (mais uniquement ?) au-dessus du bandeau qui rassemble les rubriques principales; et donc au-dessus de la rubrique « Nos collaborateurs ».

Sans aller jusqu'à l'appropriation des salariés par un pronom possessif, le site de Pernod Ricard reprend en partie cette dichotomie grammaticale entre les appartenances sociales. En effet, toutes les rubriques principales sont constituées d'un nom précédé d'un article défini : « Le groupe », « Les marques », «La RSE », 
"Les collaborateurs ». Cet usage met sans doute Pernod Ricard à l'abri d'une réification langagière de ses salariés. Cependant, il s'applique aux personnes morales et physiques qui dépendent directement de lorganisation et qui peuvent être, de facto, considérées comme soumises à un lien de subordination. A l'inverse, la rubrique destinée aux «Investisseurs » (qui par définition ne sont pas « agis » par les décisions managériales) n'est précédée d'aucun article. Il est vrai aussi, comme pour le site de Carrefour, quelle figure au-dessus du bandeau des rubriques principales.

Là encore, l'hypothèse d'une reconnaissance stratégique des salariés pourrait être émise, à l'exemple de celle pratiquée vraisemblablement par le site de Carrefour en 2013 et 2014. Cependant, le groupe Pernod Ricard n'a pas vécu de crise récente. En revanche, son histoire, sa structure capitalistique et son organisation incitent à explorer la piste d'un modèle social particulier, celui de " l'entreprise communauté ». Ce modèle se caractérise, entre autres, par une conception patriarcale et familiale du management, volontairement entretenue et maintenue tout au long d'un développement économique parfois mondial. Le fondateur est alors une " figure mythique de la communauté » dont la personnalité autorise "une relation affective entre l'entreprise et ses salariés, qui peut être qualifiée de paternelle ». Dans cet "espace de filiation » que constitue l'entreprise, le père fondateur et ses représentants assurent l'harmonie et permettent à chacun d'occuper une place, « c'est-à-dire une position dans la hiérarchie symbolique de l'entreprise " (Osty, Uhalde, Sainsaulieu 190192). La rubrique "Les collaborateurs " sur la page d'accueil du site web institutionnel du groupe Pernod Ricard assurerait donc, à chaque salarié, la reconnaissance de son appartenance pleine et entière à une communauté de destin partagé avec les quinze membres de la famille Ricard présents à des postes de responsabilité dans l'organigramme de la société (Arnaud, 2015). Certes, d'autres groupes du CAC40 sont nés d'une aventure individuelle récente (Accor, Bouygues, Capgemini, Kering, LVMH, L'Oréal, Publicis...). Mais combien allouent, dans leur structure gestionnaire et décisionnelle, une place aussi prépondérante aux héritiers et aux descendants directs de leur fondateur ? Et combien assurent, de fait, une visibilité symbolique à leurs salariés dans l'espace du web entrepreneurial ? Aucun.

L'internaute visiteur d'un site web institutionnel d'entreprise reste en grande partie un inconnu protéiforme. Au gré de sa consultation des pages-écrans, selon la nature de sa recherche et en fonction de ses appartenances sociales, il peut mobiliser des dispositions variées qui ne s'intègrent pas toujours de manière harmonieuse dans un dispositif de communication unique. $\mathrm{Ce}$ dispositif est donc l'objet d'une négociation et d'un ajustement entre les différents services et métiers qui, au sein de l'instance entrepreneuriale, s'estiment compétents pour en assurer l'implémentation, le développement, la gestion et l'animation. 
L'étude des pages d'accueil des sites web des entreprises du CAC40 confirme et complète ce constat. Elle rappelle la pesanteur institutionnelle de la communication financière qui rend incontournable la figure de l'investisseur et de l'actionnaire pour la majorité des sites. Elle montre aussi le rôle et le poids de la culture du secteur d'activité sur la façon dont les entreprises envisagent l'identité des internautes et les relations qu'elles doivent avoir avec eux. Enfin, de manière plus marginale mais néanmoins prégnante, elle souligne l'influence probable des décisions stratégiques des dirigeants sur les choix éditoriaux des communicants.

Cependant, cette étude des modalités de sélection et d'agencement des signes d'une page d'accueil doit être mise en perspective avec une évolution plus globale qui fait de la capacité à susciter et à maintenir l'attention un enjeu économique et organisationnel majeur (Kessous, Mellet, Zouinar). Or, les interfaces graphiques développées pour les écrans mobiles et tactiles ne s'accommodent guère du caractère textuel et arborescent des rubriques d'un site web 1.0. De même, le régime d'engagement des internautes dans l'exploration d'un site web institutionnel d'entreprise n'est sans doute pas déterminé, en totalité et en continuité, par les seules identités et appartenances sociales proposées. Certes, un tel site n'incite pas à la curiosité comme un site marchand ou à l'excitation comme un jeu en ligne. Mais il ne peut pas, non plus, reposer sur la seule routine d'usage d'un logiciel professionnel ou d'une base de données économiques et financières.

Dès lors, un suivi diachronique de l'évolution des pages d'accueil permettrait de pondérer l'importance et l'influence des changements technologiques face à l'épaisseur du social. De même, une approche monographique fondée sur différentes méthodes d'enquête (observation participante, étude des documents prescripteurs, entretiens approfondis...) permettrait d'analyser les critères de choix et les mécanismes de décision qui conduisent les entreprises à considérer la page d'accueil de leur site web institutionnel comme digne d'intérêt et de considération.

\section{BIBLIOGRAPHIE}

ARNAUD Jean-François (2015), «Un Ricard sinon rien... même à la tête de l'entreprise familiale ", site de ChallengeSoir, disponible sur www.challenges.fr/entreprise/20150211.CHA2981/un-ricard-sinon-rien-memea-la-tete-de-l-entreprise-familiale.html, consulté le 15 octobre 2015.

D'ALMEIDA Nicole (2006), « La perspective narratologique en organisation », dans P. de la Broise et Th. Lamarche, Responsabilité sociale : vers une nouvelle communication des entreprises? Villeneuve d'Ascq, Septentrion, 27-38.

BERNARD Françoise (2015), « La communication des organisations entre questions d'influence et questions d'autonomie. L'actualité des notions d'engagement, d'émergence et d'institution ", In Communication et organisation, n ${ }^{\circ} 47$, p. 85-95. 
BIBLIOTHÈQUE NATIONALE DE FRANCE, Archives de l'internet.

BOCQUET Anaïs, LACHAUD Gérald (2013), « Les figures sociétales du client mobilisées par la communication électronique des marques. Le cas des plates-formes participatives Web ", Dans S. Zlitni et F. Liénard, La communication électronique en questions, Bern : Peter Lang, p. 21-36.

BOUCHER Amélie (2015), Expérience utilisateur mobile. UX design pour smartphones et tablettes, Paris, Eyrolles, 286 p.

CAPRON Michel, QUAIREL-LANOIZELÉE Françoise (2004), Mythes et réalités de l'entreprise responsable. Acteurs, enjeux, stratégies, Paris, La Découverte, $252 \mathrm{p}$.

CHAUVIÈRE Michel, GODBOUT Jacques T. et al. (1992), Les usagers entre marché et citoyenneté, Paris, L'Harmattan, 332 p.

COCHOY Franck (1999), Une histoire du marketing. Discipliner l'économie de marché, Paris, La Découverte, 391 p.

COCHOY Franck (2004), « La captation des publics entre dispositifs et dispositions, ou le petit chaperon rouge revisité ", dans F. Cochoy, La captation des publics. C'est pour mieux te séduire, mon client..., Toulouse, Presses Universitaires du Mirail, 11-68.

DAVALLON Jean, JEANNERET Yves (2004), " La fausse évidence du lien hypertexte ", In Communication et langages, $\mathrm{n}^{\circ} 140$, p. 43-54.

FELDMAN Regina M., FELDMAN Steven P. (2006), "What Links the Chain : An Essay on Organizational Remembering as Practice ", in Organization, n 13(6), p. 861-887.

GARDÈRE Elizabeth, GRAMACCIA Gino (2003), «Anthropologie des petits groupes : de Lewin à Midler. Une brève histoire de la coopération dans les collectifs restreints ", dans E. Gardère et G. Gramaccia, Coexister dans les mondes organisationnels, Paris, L'Harmattan, 99-141.

GERVAIS Michel, HERRIAU Christophe (2011), Stratégie de l'entreprise, Paris, Economica, $6^{\mathrm{e}}$ éd., $543 \mathrm{p}$.

GIMELLO-MESPLOMB Frédéric, VILATTE Jean-Christophe (2015), « Les recherches sur les publics en sciences de l'Information et de la Communication ", in Revue française des sciences de l'Information et de la Communication, $\mathrm{n}^{\circ} 7$, site de revues.org, disponible sur http://rfsic.revues.org/1466, consulté le 14 octobre 2015.

GOFFMAN Erving (1968), Asiles. Études sur la condition sociale des malades mentaux, Paris, Les Éditions de Minuit, 449 p.

GRAMACCIA Gino (2001), Les actes de langage dans les organisations, Paris, L'Harmattan, 287 p.

HELLER Thomas (2009), "Reconnaissance et gouvernement des salariés. Au-delà du mépris ", in Questions de communication, $\mathrm{n}^{\circ}$ 15, p. 93-107.

HETZEL Patrick (2002), Planète conso: marketing expérientiel et nouveaux univers de consommation, Paris, Ed. d'Organisation, 380 p.

HONNETH Axel (2006), La société du mépris. Vers une nouvelle théorie critique, Paris, La Découverte, 349 p. 
HUREL du CAMPART Sabine, AACC corporate (2003), La communication corporate. Enjeux et acteurs (entreprises/agences) de la communication des entreprises, Paris, Dunod, 209 p.

KESSOUS Emmanuel,MELLET Kevin,ZOUINAR Moustafa (2010), «L’économie de l'attention : entre protection des ressources cognitives et extraction de la valeur », In Sociologie du travail, $\mathrm{n}^{\circ}$ 52, p. 359-373.

LÉGER Jean-Yves (2008), La communication financière, Paris, Dunod, 278 p.

LEGRIS-DESPORTES Christiane (2011), "Approche socio-sémiotique de la relation client ", In Communication et organisation, $\mathrm{n}^{\circ}$ 39, p. 125-136.

LE MOËNNE Christian (2006), «Les communications d'entreprise entre médias, réseaux et recompositions organisationnelles ", dans A. Bouzon, La communication organisationnelle en débat : champs, concepts, perspectives, Paris, L'Harmattan, p. 103-121.

LIBAERT Thierry, de MARCO André (2006), Les tableaux de bord de la communication. Indicateurs de pilotage et évaluation des résultats, Paris, Dunod, $246 \mathrm{p}$.

LIBAERT Thierry (2013), La plan de communication. Définir et organiser votre stratégie de communication, Paris, Dunod, $4^{\mathrm{e}}$ éd., 270 p.

MEIGNAN Géraldine (2013), "Georges Plassat, le patron de Carrefour qui parle "cash"», site de l'Expansion/l'Express, disponible sur http://lexpansion.lexpress.fr/ entreprises/georges-plassat-le-patron-de-carrefour-qui-parle-cash_1412259. html, consulté le 7 juillet 2014.

MIÈGE Bernard (1995), «L'espace public contemporain: perpétué, élargi et fragmenté », dans I. Pailliart, L'espace public et l'emprise de la communication, Grenoble, Ellug, p. 163-175.

OSTY Florence, UHALDE Marc, SAINSAULIEU Renaud (2007), Les mondes sociaux de l'entreprise : penser le développement des organisations, Paris, La Découverte, 398 p.

REVUE DU DIGITAL, 2014, "Carrefour se refonde tout en travaillant à son projet multi-canal centré sur le mobile ", site de La Revue du digital, disponible sur http://www.larevuedudigital.com/2014/06/11/carrefour-se-transforme-tout-entravaillant-a-son-projet-multi-canal-centre-sur-le-mobile/, consulté le 17 septembre 2015.

ROUQUETTE Sébastien (2009), L'analyse des sites internet. Une radiographie du cyberespace. Paris/Bruxelles, INA/De Boeck, 335 p.

RYAN Gerard, GUBERN Marcel, RODRIGUEZ Inma (2000), « Recruitment advertising : the Marketing-human ressource interface ", in International Advances in economic research, $\mathrm{n}^{\circ} 2$, p. 354-364.

SCAPIN Dominique, SENACH Bernard, TROUSSE Brigitte, PALLOT Marc (2012), "User Experience: Buzzword or New Paradigm?», ACHI 2012, The Fifth International Conference on Advances in Computer-Human Interactions, January 30 - February 4, Valencia, Spain, site du projet Orion-Inria, disponible sur https://www.thinkmind.org/download.php?articleid=achi_2012_13_40_20153

STEIN Eric W. (1995), "Organizational Memory : Review of Concepts and Recommendations for Management », in International Journal of Information Management, 15(2), p. 17-32. 
VITALIS André et al. (1994), Médias et nouvelles technologies. Pour une socio-politique des usages, Rennes, Éditions Apogée, 159 p.

WEICK Karl E. (1995), Sensmaking in Organizations, Thousand Oaks, Sage Publications, 248 p.

Résumé : Cet article questionne la place de l'identité sociale des internautes dans les rubriques de la page d'accueil du site web institutionnel des entreprises du CAC40. À l'appui de ce corpus, il montre les différents facteurs qui conduisent les firmes à privilégier certaines identités : poids de la communication financière, contexte économique, juridique et médiatique, influence du secteur d'activité, décision stratégique des dirigeants.

Mots-clés : communication, entreprise, web, identité sociale, page d'accueil.

Abstract: From an analysis of Web homepage, this article examines the issue of social identities in the corporate digital communication of major French companies. It shows the influence of the financial purposes. But, customer relationship and stategic choices could also explain the presence of social identities in the homepage of a Web site.

Keywords : corporate communication, web homepage, social identity. 